\title{
Assessment of the independent work efficacy of natural sciences students
}

\author{
Anna Fomina 1,*, Oxana Kundupyan ${ }^{2}$, Mikhail Bibov ${ }^{3}$, Artem Starostin ${ }^{2}$, Julia Kundupyan ${ }^{2}$, \\ and Eugeny Aydarkin ${ }^{2}$ \\ ${ }^{1}$ Don State Technical University, sq. Gagarina, 1, Rostov-on-Don, 344003, Russia \\ ${ }^{2}$ Southern Federal University, Bolshaya Sadovaya str. 105/42, Rostov-on-Don, 344006, Russia \\ ${ }^{3}$ Rostov State Medical University, Nahichevansky av.29, Rostov-on-Don, Russia
}

\begin{abstract}
The present article describes an approach for assessment of efficacy of independent work of students, studying natural sciences, using the digital technologies. Tasks were fulfilled in the Moodle electronic medium and consisted of answering the questions of the closed-type questionnaire, as well as writing answers to the questions of the Tests. We assessed time required for a student to write the answer to one question, number and correctness of the terms used and the level of plagiarism. These parameters were considered to be the effective markers to assess the level of student's knowledge during the standard test fulfillment. Key words: computer-based testing, independent work efficacy, test, terms, plagiarism level.
\end{abstract}

\section{Introduction}

Transfer to the remote studying using the digital technologies is connected with asynchronic learning, increased communication with electronic educational systems and decreased level of communication with the tutor [1,2]. Educational technologies, which provide independent work of students, are based upon the online task representation, registration of the tutor activity and automatic calculation of successfulness and efficacy of the studying. The possibility of contactless assessment of the structure of studying activities plays an important role $[3,4,5]$.

Use of electronic educational technologies leads to the development of skills required for the independent work of students, increases their motivation to reach the successful results and sustains the effectiveness of the studying process [4,6,7]. This results in the formation of individual studying strategy. Successful independent work allows students to adapt easily not only to the conditions of educational process, but also to the real professional work $[7,8]$.

The complexity of assessment of the independent work of students is due to poor formalization of the assessment criteria. This is especially true for the tasks, which require the text report writing. The main role belongs to the assessment of a student's knowledge using the standardized tests and situational tasks [4,9]. Besides general effectiveness of the study, the registration of behavioral parameters, such as time a student needs need to answer

*Corresponding author: a_bogun@mail.ru 
one question of the open and closed tests, quality of work and the number of switches between them $[10]$ is also required $[3,7,11,12]$.

In the present study we assessed the efficacy of independent work of students during the fulfillment of standard studying tasks.

\section{Materials and methods}

In the present work we assessed the knowledge control methods used in the courses of "Human Anatomy" and "Particular Physiology of Central Nervous System", in which 24 second-year students participated. Students were asked to fulfill both theoretical and practical tasks of following types:

1. Closed tests: the block of tasks, which contained the closed type questions. To fulfill this tasks, students were asked to choose the correct answer among the proposed ones. Each question included at least four variations of the answer. Each test included at least 25 questions, which were chosen randomly for each student.

2. Open tests: the block of tasks, which contained both theoretical and empirical questions, the answer to which implied writing of a short essay, work with images and making conclusions from a simplified scientific situation. Each open test included $7-10$ questions. This part of work required use with scientific literature and was accomplished with a theoretical conclusion.

Work with the closed and open tests was carried out in the special medium with nonlinear editing in the Moodle educational system. This system provided automatic assessment of the number of correct answers during answering to the closed tests, taking into account the critical border, which was set by the tutor. Also, in the Moodle system the tutor checked the results of the open tests and gave marks.

To perform the analysis, we considered 88 study sessions; the duration of each session was $3 \mathrm{~h}$. For the fulfillment of open tests, students were awarded with marks in accordance with the 5-grade system and results of the tutor's expert assessment. Fulfillment of the closed tests was evaluated by the percentage of correct answers, which were detected automatically by the Moodle system. Quality of the closed and open test fulfillment, time required to answer one question, the number of terms used, literacy and the level of plagiarism were analyzed. Artefactless sections of EEG were used to analyze the spectral power. Confidence of differences between the parameters was assessed by ANOVA, $\mathrm{p} \leq 0.05$.

\section{Results}

All students were divided into the successful and unsuccessful ones in accordance with the results of the open test fulfillment and expert assessment of the tutor. In the group of unsuccessful students, the average academic mark for the fulfillment of the open tests in the 5 -grade system was 1,8 80,14 ( 0 - 2.5 grades). In this group, some students either refused to fulfill the educational task or provided an extremely short answer. In the group of successful students, the average academic mark in the 5-grade system was 3,9 $\pm 1,0$ (3.5 - 5 grades) and was confidently higher as compared with the unsuccessful group $(1: 1, F=21,2, p=0,001)$. The Fig. 1 shows the ratio of academic progress of the students. 


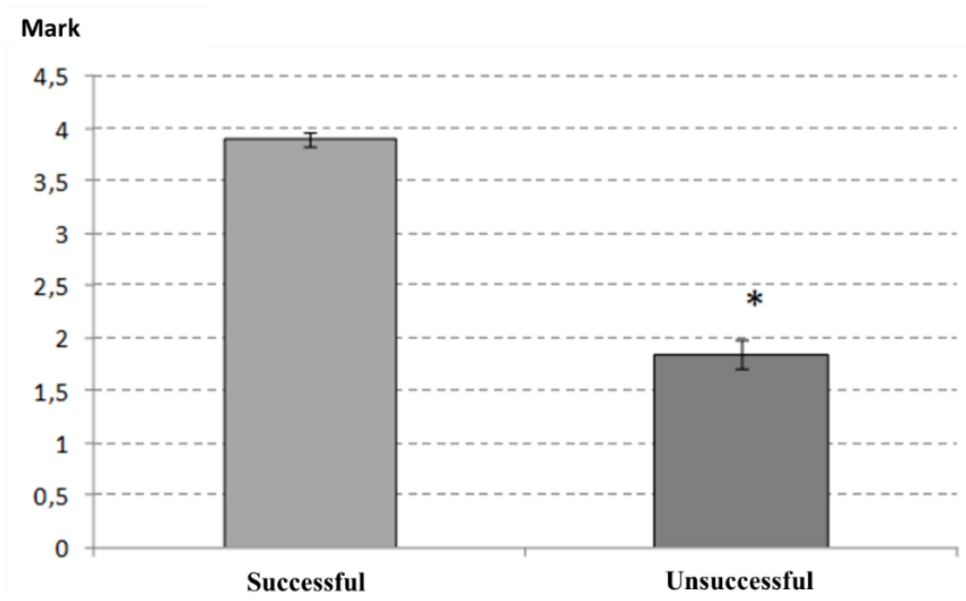

Fig. 1. Average grades of students in the groups with different academic progress. Symbols: the abscissa shows the groups of students; ordinate shows the academic grades. "*" indicates confident differences.

The Fig. 2 shows the average time required to answer one question of the open test. It is shown that a successful answer took 7.12 min that was confidently longer in comparison with an unsuccessful one ( $5 \mathrm{~min})(1: 1, \mathrm{~F}=9,8, \mathrm{p}=0,0012)$. The group of unsuccessful students demonstrated positive correlation between the time required to answer one question of the open test and the academic mark $(r=0,4)$. However, no confident correlation between these parameters was observed in the group of successful students.

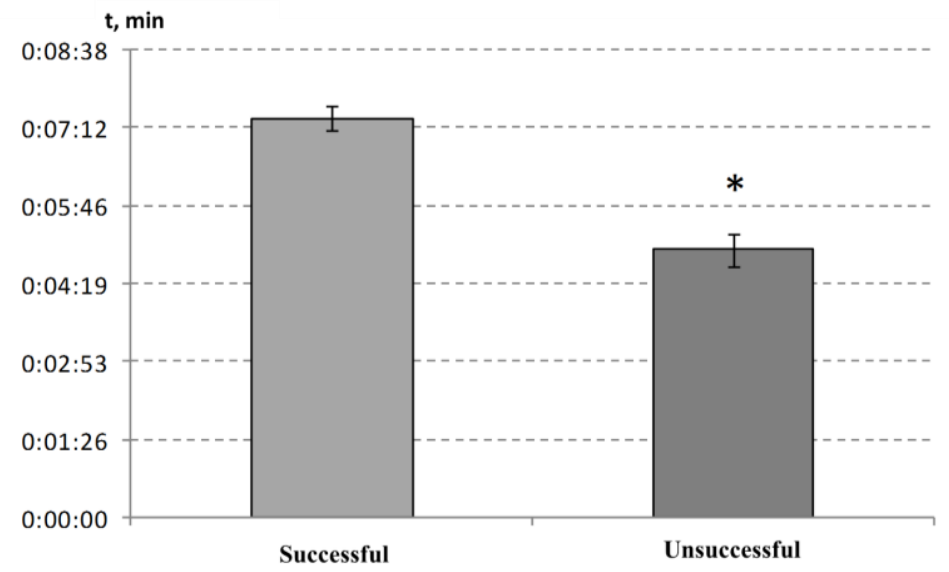

Fig. 2. Average time required to answer one question of the open test in two groups of students with different academic progress. Symbols: the abscissa shows the groups of students; ordinate shows the time required for the answer, s. "*" indicates confident differences.

Hence, the analysis of the quality of task solving showed that the successful students managed with the task at the "perfect" level, and it did not take them long to fulfill the test. The successful students also demonstrated a big number of correct answers to the tasks, which required an essay writing, as well as the work with anatomic images and schemes. unsuccessful students demonstrated low quality of solving of the tasks of both types. Rather often they even refused to do the work at all. A part of unsuccessful students demonstrated good results in the essay writing though dealt worse with analysis of images (sometimes they did not give the answer at all). 
Further analysis revealed that these groups of students differed from each other by the number of professional terms used in the answers. The number of terms used was assessed as the percentage of the terms used from their number set by the tutor. The Fig. 3 shows the percentage of this parameter in both groups. It was shown that the successful students used, on an average, $74,5 \pm 2,7 \%$ of terms. Unsuccessful students used far less terms $(52,5 \pm 2,9 \%)$ $(1: 1, F=32,564, p=0,0035)$.

Therefore, the portion of the terms used in a successful answer was 1.5-times higher with a strong positive correlation $(r=0,7)$ with the response time. Successful students did not do mistakes in the term writing, whereas unsuccessful students did it from time to time.

Moreover, the group of successful students demonstrated weak negative correlation between the time required to answer one question of the open test and the portion of terms used $(r=-0,38)$. Conversely, the group of unsuccessful students was characterized by medium positive correlation between these parameters $(r=0,53)$.

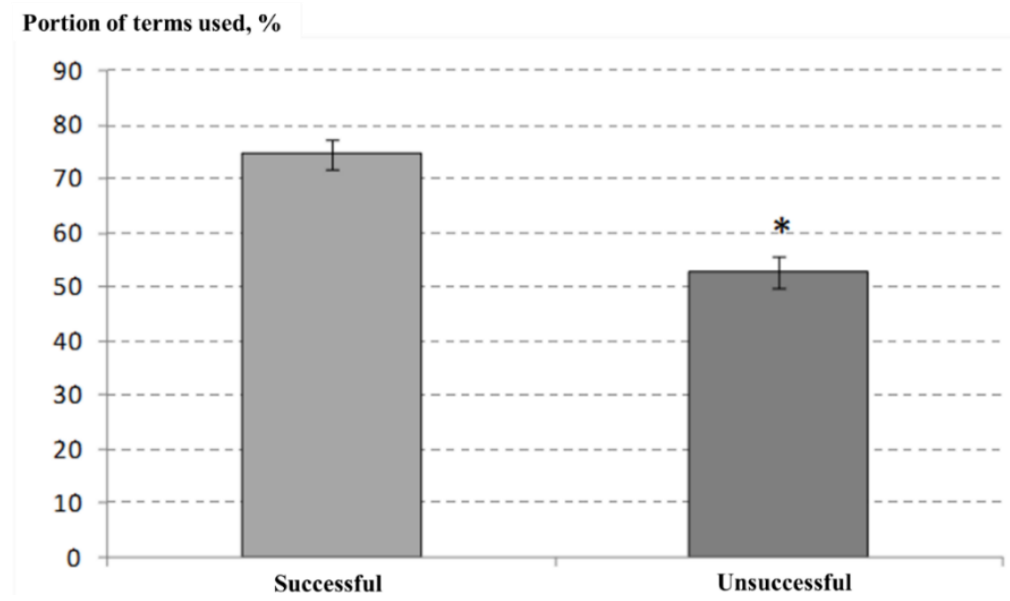

Fig. 3. The average portion of special terms used by the students in the written answer to one question of the open test in two groups with different academic progress. Symbols: the abscissa shows the groups of students; ordinate shows the portion of terms used, \%. "*" indicates confident differences.

This may be, apparently, explain as follows. The successful students sometimes tried to substitute a specialized term by a general scientific expression. This does not distort the content of the answer and was not considered as a misuse of terminology, as it demonstrated that the student understands the principles of a biological process or event. However, choice of a correct synonym increased the time of the answer to one question. The converse dependence in the group of unsuccessful students showed the absence of efforts to choose synonyms of the terms and provide explanations, which would operate with general scientific terms.

The assessment of the plagiarism level was performed with the "Antiplagiat.VUZ" program. The successful students demonstrated low level of plagiarism $(9,2 \pm 2 \%)$, which was due to the use of standard expressions and complex terms. In the group of unsuccessful students, the search of information implied the efforts to find the full-text and ready-to-use answers. Hence, the percentage of the word-for-word borrowings made up $50 \%$ or more in this group.

The successful students were characterized by full and unmistakable use of special terms, longer period of answering to one question of the open test and absence of a significant level of plagiarism.

Students, who demonstrated low quality of the open test fulfillment, showed poor results in the closed tests as well. Similarity in the level of an academic grade obtained for two 
different types of the control work may be considered as a criterion of the level of selfpreparation. In the group of unsuccessful students, the average progress in the closed tests was $56,7 \pm 1,74 \%(32-68 \%)$, whereas in the group of successful students it was $84 \pm 1,2 \%(72-$ $96 \%)(1: 1, F=5,393, p=0,01)$. The Fig. 4 shows the progress of students in the closed tests.

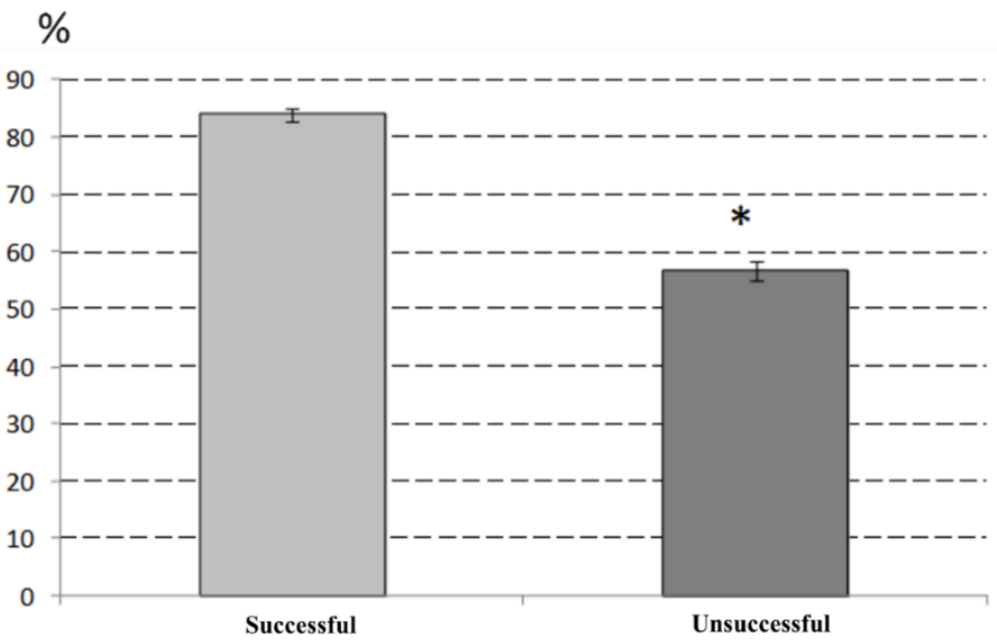

Fig. 4. Average values of successfulness of the test fulfillment in two groups of students. Symbols: the abscissa shows the groups of students; ordinate shows the portion of the correct answers, \%. "*" indicates confident differences.

The Fig. 5 demonstrates the average time required for a student to answer one question of the test. It was shown that the successful answer took $55 \pm 3 \mathrm{~s}$ that was confidently longer than that for the unsuccessful answer $(34 \pm 3 \mathrm{c})(1: 1, \mathrm{~F}=18,54, \mathrm{p}=0,03509)$. The group of unsuccessful students demonstrated negative correlation between the time required to choose the correct answer for the test question and general successfulness of the task fulfillment ( $r=-$ $0,54)$. The successful students, conversely, demonstrated low positive correlation between these parameters $(r=-0,41)$.

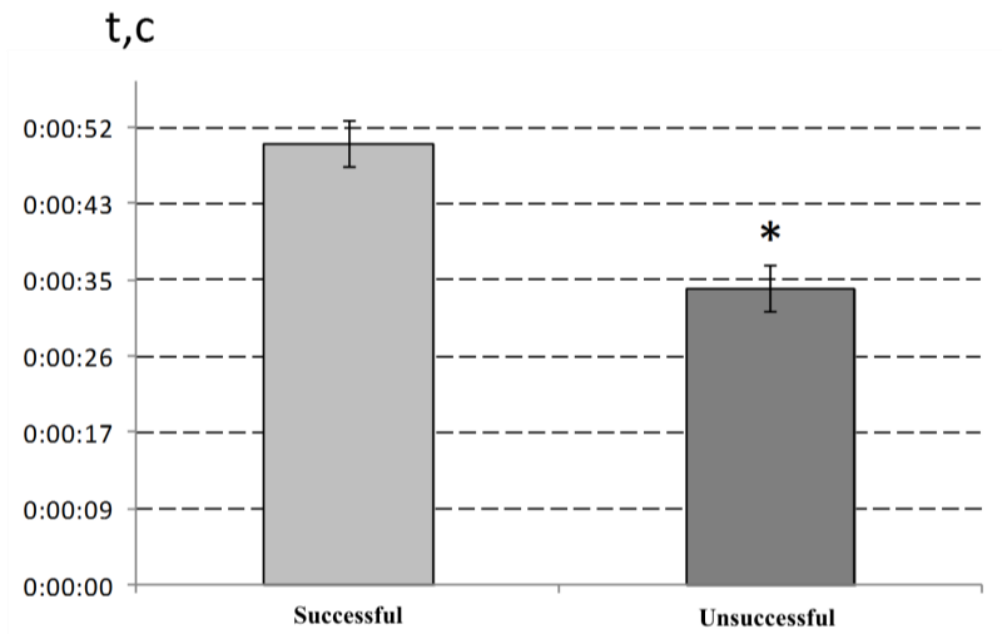

Fig. 5. Average time requires to choose the answer for one question of the test in two groups of students with different academic progress. Symbols: the abscissa shows the groups of students; ordinate shows time requires to choose the answer variation, s. "*" indicates confident differences. 


\section{Discussion}

It was shown that the dynamics of the parameters of efficacy of educational activities revealed in the present study was similar to that obtained previously under model conditions of educational process in a psychophysiological experiment [10].

Unsuccessful students demonstrated faster choice of the answer variation during the test fulfillment because of poor knowledge of special terminology. Taken together with low quality of the task solving, this allows us to suggest that these students simply tried to guess the correct answer. Longer period of the task solving by the successful students suggests careful analysis of the task proposed and writing of an answer, providing the effective activity.

The analysis of time required to answer individual questions of the open tests revealed converse situation. This is also due to knowledge of terminology. Unsuccessful students wasted small time to write the essays of the open tests, because of poor knowledge of the subject studied. This prevented them from writing of a full-text answers, analyzing and generalization of the biological problem proposed and making conclusions from given model tasks and practical data. Therefore, these students failed to answer many of the open task questions. Conversely, the successful students, who became proficient in the subject of study because of careful self-preparation, provided full-text and detailed answers to the open test questions with low level of plagiarism.

Apparently, the revealed differences in general mastering of the information studied and, particularly, study of special terminology may explain the converse dynamics in time required to fulfill the blocks of tasks and answer individual questions of closed and open tests in the groups with different academic progress.

This allows us to suggest different strategy of activity in these groups of students. The questions being formulated quire clearly and shortly, the formulation of an answer required good knowledge of theoretical content of the subject. Low mark was followed by short time wasted to write the answer, which was much shorter than that of the students with high academic mark. This approach to the educational task fulfillment was acknowledged as the least effective one. Apparently, in this case, low level of self-preparation of students outside of the classes was followed by low level of motivation to the task solving. High efficacy of academic activity was associated with long time required to write an answer to a question of the open test and short time required to choose an answer in the closed test, whereas low quality of the task solving was connected with opposite dynamics.

Hence, one may suggest that successful task solving implies more active and successive involvement of cognitive processes, such as, first of all, volitional attention, in order to manage with complex intellectual tasks [7,13-15]. The dependencies observed may be smoothed by perfunctory activities, which are carried out with low motivation and lack of time.

It is noteworthy that fulfillment of the closed test tasks required different time under model and real educational conditions. In model experiments, longer period of answering of one of the closed test questions was observed in case of unsuccessful task solving, whereas in real educational process it was, conversely, typical for successful students. Both factors may be explained by unsatisfactory knowledge of special terms of unsuccessful students that forced them to switch for the guessing of answers for the test and efforts to recall the term meanings in the model experiment. 


\section{Conclusions}

In results of our study brought us to the conclusion that the parameters of time required to write an answer to one question, number and correctness of the terms used and the level of plagiarism may be used as the markers to assess the level of student's knowledge during the test solving. The correct use of special terms allows not only assess the level of proficiency in a discipline, but also to estimate analytic skills, capacity to generalization of a concept and to use in correctly in order to solve model tasks. Differences in the rate of the test task solving in the groups of students with different academic progress may be also explained by different capacity to correct use of terms.

\section{References}

1. K. Lyons, J.E. McLaughlin, J. Khanova, M.T. Roth, Adv. Health Sci Educ Theory Pract., 22(3), 723-739 (2017)

2. L. Heidi, A. Raizada, S.E. DiCarlo, Adv Physiol Educ. 45(1), 59-60 (2021)

3. I.C. Chahine, Journal of Mathematical Behavior 32, 434-449 (2013)

4. J.P. Kim, S.W. Seo, H.Y. Shin et al., Neurology 85(9), 806-812 (2015)

5. S.J. Ritchie, T.C. Bates, I.J. Deary, Dev Psychol. 51(5), 573-582 (2015)

6. C. Desmet, I. Imbo, J. De Brauwer et al., Q J Exp Psychol. 65(6), 1059-1067 (2012)

7. J. Rodrigues, N.C. Jordan, N. Hansen, Journal of Learning Disabilities 52(6), 480-497 (2019)

8. M.A. D'Eredita, W.J. Hoyer, Experimental Aging Research 36, 23 -39 (2010)

9. J.M. Phillips, J Contin Educ Nurs. 36(2), 77-83 (2005)

10. A. Fomina, O. Kundupyan, A. Starostin, M. Bibov, E3S Web of Conferences 210, 18089 (2020)

11. E.K. Aydarkin, A.S. Fomina, Journal of Integrative Neuroscience 12(1), 73-89 (2013)

12. E.K. Aydarkin O.L. Kundupyan, J.L. Kundupyan, Journal of Integrative Neuroscience 12(1), 57-72 (2013)

13. W. Skrandies, A. Klein, Brain research 1603, 133 -145 (2015)

14. W. Van Dooren, E. Lehtinen, L. Verschaffel, Learning and Instruction 37, 1-4 (2015)

15. M.T. van Kesteren, M. Rijpkema, D.J. Ruiter et al., J CognNeurosci. 26(10), 2250-2261 (2015) 